\title{
Remote evaluation of video-otoscopy recordings in an unselected pediatric population with an otitis media scale
}

\author{
Thorbjörn Lundberg ${ }^{a, *}$, Leigh Biagio ${ }^{\mathrm{b}}$, Claude Laurent ${ }^{\mathrm{b}, \mathrm{c}}$, Herbert Sandström ${ }^{\mathrm{a}}$, \\ De Wet Swanepoel ${ }^{\mathrm{b}, \mathrm{d}, \mathrm{e}}$ \\ a Department of Public Health and Clinical Medicine, Family medicine, Umeå University, S-901 87 Umea, Sweden \\ ${ }^{\mathrm{b}}$ Department of Speech-Language Pathology and Audiology, University of Pretoria, Pretoria, South Africa \\ ${ }^{\mathrm{c}}$ Department of Clinical Sciences, Otorhinolaryngology, Umeå University, Umea, Sweden \\ d Ear Science Institute Australia, Subiaco, Australia \\ e Ear Sciences Centre, School of Surgery, The University of Western Australia, Nedlands, Australia
}

Otitis media

Grading

Tympanic membrane

Video-otoscopy
A B S T R A C T

Background: A recently validated image-based grading scale for acute otitis media (OMGRADE) can be used to assess tympanic membrane (TM) status. The aim of this study was to evaluate the validity and reliability of this scale for remote assessments of TM status using video-otoscopy recordings in an unselected pediatric population.

Method: Children 2-16 years attending a South African primary health clinic were offered an ear examination by an otologist using otomicroscopy. An ear and hearing telehealth facilitator then made video-otoscopy recordings (9-33 s) of the ears and uptakes were uploaded to a secure server for remote assessments in Sweden by an otologist and general practitioner at four- and eight-weeks post onsite assessment. TM appearance was judged according to the OMGRADE scale. Concordance between onsite otomicroscopy and asynchronous assessments of video-otoscopy recordings was calculated together with intra- and inter-rater agreements.

Results: One hundred and eighty ears were included. Concordance of TM classifications using the OMGRADE scale was found to be substantial (weighted kappa range 0.66-0.79). Intra- and inter-rater agreement (test-retest) was found to be substantial to almost perfect (weighted kappa range $0.85-0.88$ and $0.69-0.72$, respectively).

Conclusion: The OMGRADE scale can be used to accurately assess the normal TM and secretory otitis media (SOM) remotely using video-otoscopy recordings in an unselected pediatric population.

\section{Introduction}

Middle ear infection - otitis media - is among the most common infections in children [1]. The burden of otitis media differs between developed and developing countries with the incidence of acute otitis media (AOM) in sub-Saharan Africa, South Asia and Oceania reported to be two to eight times higher than in other regions of the world [1]. In addition, the lack of sufficient numbers of specialists, such as otolaryngologists, family physicians and audiologists, to serve the majority of populations around the world [2] necessitate new approaches to overcome these discrepancies. In this context telemedicine may be able to offer remote,

\footnotetext{
* Corresponding author. Tel.: +46 9078500 00; fax: +46 90776883 . E-mail address: thorbjorn.lundberg@fammed.umu.se (T. Lundberg).
}

highly specialized clinical assessments to such underserved areas [3].

Otitis media is a group of different diagnoses including otitis media with effusion (OME), acute otitis media (AOM) and chronic suppurative otitis media (CSOM). To diagnose the various forms of otitis media and their respective stages require an assessment of the tympanic membrane (TM) using either otoscopy, otomicroscopy or, more recently, video-otoscopy with still images or recordings [4]. It is also necessary to use a standardized grading system for the TM appearance in order to classify and grade the disease and its different stages [5,6]. Using a standardized grading scale for otitis media may allow for more comparable assessments of the TM and otitis media diagnoses with less clinical variability.

Different scoring systems and a single AOM grading scale have previously been presented [7-11]. However, in our opinion, the aforementioned systems tend to overestimate the value of the 
Table 1

Different ratings of tympanic membrane status at all assessments.

\begin{tabular}{|c|c|c|}
\hline \multicolumn{2}{|l|}{ Label } & \multirow[t]{2}{*}{ Description } \\
\hline OMGRADE & Sub-division & \\
\hline 0 & 0 & Transparent TM, normal position \\
\hline \multirow[t]{3}{*}{1} & $1 \mathrm{R}$ & Transparent TM, slightly retracted \\
\hline & $1 \mathrm{~F}$ & Transparent TM, normal position, fluid level or fluid filled ME \\
\hline & $1 \mathrm{RF}$ & Transparent TM, retracted with fluid level or fluid filled ME \\
\hline 2 & $2 \mathrm{OF}$ & Transparent TM with opaque fluid level, w/wo retraction \\
\hline 3 & 3 & Opaque appearance of TM in a fairly normal position \\
\hline 4 & 4 & Opaque appearance of TM and bulging \\
\hline \multirow[t]{2}{*}{5} & $5 \mathrm{~B}$ & Opaque appearance of TM with bullous formations \\
\hline & $5 \mathrm{C}$ & Contourless TM with a wet appearance and swollen keratin patches, w/wo pulsating pus from small perforation \\
\hline
\end{tabular}

Temporary subgrade

6

TM perforation, retraction pocket or cholesteatoma w/wo purulent discharge, previous ear surgery and TM grommets

Not possible to determine NPD

Not possible to determine due to obscuring objects, low image quality or inability to inspect the entire TM-surface

Abbreviations: ME: middle ear; w/wo: with or without; NPD: not possible to determine; VO: video-otoscopy.

parameter "redness of the TM" despite colour having been found to be of limited value in diagnosing AOM $[11,12]$. In order to improve the grading of AOM and to follow the course of the disease over time, a validated image-based grading scale for AOM (OMGRADE scale) was recently developed [13]. It includes different stages seen during the course of AOM, from the normal TM to the pathological TM's in various stages of AOM (Table 1). However, the present OMGRADE scale does not include ears with CSOM.

The OMGRADE scale should be applicable to many clinical situations including telemedicine contexts where a standardized grading system for otitis media can serve as a diagnostic guide for evaluating still TM images or video recordings of TM's remotely, together with evaluation of middle ear effusion by tympanometry or pneumatic video-otoscopy. A study conducted in rural Australia indicated that good quality endoscopic still images of the TM were sufficient for adequate clinical otological diagnosis [14]. Videootoscopy, utilizing still images, has been shown to have a high sensitivity and specificity as compared to pneumatic otoscopy and tympanometry in evaluating OME [15]. Combining TM images or video recordings of TM's with new systems for hearing assessments in a telemedicine setting could provide a valuable diagnostic tool for rural and underserved areas in the world [4,16].

A recent study has demonstrated that a general ear and hearing telehealth facilitator (EHTF) can be trained to acquire video-otoscopic images for remote diagnosis by professionals (otolaryngologists and general practitioners) from different parts of the world [17].

The aim of this study was to evaluate the validity and reliability of the OMGRADE scale for remote assessment of TM-status using video-otoscopy recordings (video clips) in an unselected pediatric population.

\section{Method}

\subsection{Study population}

This consecutive study was conducted following approval from the Institutional Ethics Committee at the University of Pretoria, Pretoria, South Africa.

A sample of 140 children aged 2 to 16 years (range 2-15.8 years, mean age $6.4 \pm 3.5$ years, $44.4 \%$ females) were recruited during a two week period from the entire pediatric population attending a primary health care clinic, irrespective of reason for attendance. The Witkoppen Health and Welfare Centre provides health care services to poor populations, including the Diepsloot community north of Johannesburg. Diepsloot is a densely populated settlement made up of government subsided brick houses and shacks. Unemployment exceeds $90 \%$, and the access to basic services such as running water, sewage and rubbish removal is limited [18]. After verbal and written information, caregivers were required to provide informed consent before any data collection was started. Caregivers and children were then interviewed immediately before examination to obtain biographical information and history of any earache, ear discharge or hearing loss during a two-week period prior to the participation in the study and the data were recorded.

\subsection{Otomicroscopy}

Otomicroscopy was performed for each ear by an experienced (>35 years of practice) otologist using a Leica M525 F40 surgical otomicroscope with a 6:1 zoom magnification $(1.2-12.8 \times)$ and a $300 \mathrm{~W}$ xenon fibre optic illumination. Cerumen was manually
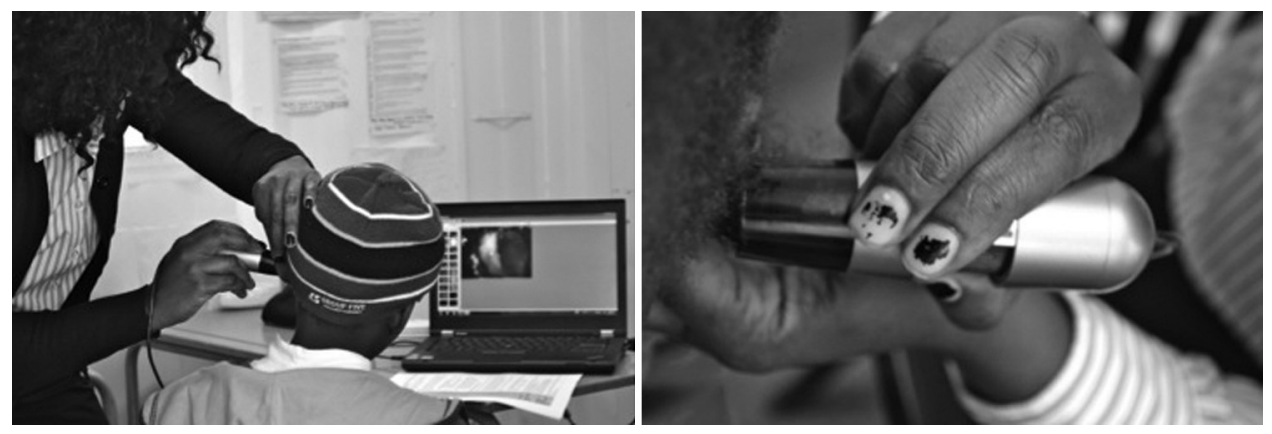

Fig. 1. Ear and hearing telehealth clinic facilitator documenting TM status with video-otoscopy. 
removed when necessary in order to obtain an acceptable view of the TM for diagnosis. Ears with obstructing wax that was not possible to remove without discomfort were excluded from further analyses in the study and the numbers recorded.

The diagnosis based on otomicroscopy was set using both a classification of otitis media in one of the three groups AOM, OME or CSOM [19] and with the OMGRADE-scale. AOM was based on the finding of a bulging and opaque TM or with the finding of middle ear effusion and mild bulging in a child with rapid onset of othalgia or fever. AOM was also set if there was a wet and contourless perforated TM (chagrinated). The OME was based on the finding of fluid levels or a completely opaque and non-bulging TM in a child without signs of an acute infection. The diagnosis CSOM in this study was based on a visible TM perforation, deep retraction pocket or cholesteatoma with or without purulent discharge, and previous ear surgery or grommets present in the TM.

TM status was also classified according to the OMGRADE scale [13]. This newly validated image based grading scale was originally constructed with 6 basic steps $(0-5)$ for different stages in the course of AOM (Table 1 ). Steps 1 and 5 were divided into "sub grades". However, the OMGRADE can also be used as classification tool for otitis media. A transparent TM in a normal position or slightly retracted is diagnosed as normal and OMGRADE scale step 0 or $1 \mathrm{R}$. The OMGRADE scale steps and sub grades $1 \mathrm{~F}, 1 \mathrm{RF}, 2 \mathrm{OF}$ and 3 would all fall within a traditional OME diagnosis (slightly retracted ear drum with or without various amount of serous fluid, opaque fluid or a non-transparent TM in a fairly normal position). Neither tympanometry nor pneumatic otomicroscopy was available at the clinic. The OMGRADE scale steps are possible to compare with the respective diagnoses of AOM and OME. Although the present OMGRADE scale does not include ears with CSOM, a temporary grade $6(t-6)$ for ears with CSOM was incorporated in the OMGRADE for the current study.

The otologist examined each TM otomicroscopically, made a diagnosis and provided an OMGRADE rating in scale steps $0-5$ with subgrades, including t-6 (Table 1). The onsite otomicroscopic examination by the otologist was considered the gold standard diagnosis.

\subsection{Video-otoscopy recordings}

Directly following otomicroscopy the children underwent videootoscopy recordings made from each ear. An EHTF, who had no formal health care or tertiary education, made the video-otoscopic recordings (Fig. 1). For a two-day period prior to the study the otologist carried out onsite training of the EHTF on how to conduct the video-otoscopic recordings. The child was positioned in a stable armchair or in the caregiver's lap watching the screen of a laptop connected to a video-otoscope. The EHTF was positioned beside the child on the side of the ear to be examined (Fig. 1).

The video-otoscopic recordings were made with a Dino-Lite Pro Earscope (USB) with a LED light, a magnification of $10-20 \times$, a frame rate of 30 frames/s and a $1.3 \mathrm{MP}$ resolution. The Dino-Lite video-otoscope was attached via a USB cable to a Lenovo ThinkPad 2.0 running Windows 7 via 2.0 interface or a Macbook pro running OSX v10.7.5. DinoCapture 2.0 software (AnMo Electronics Corporation) version 1.2.7 was used to record and view the videootoscopic recordings. Depending on the size of the external ear canal a 3,4 or $5 \mathrm{~mm}$ speculum was attached on the video-otoscope head. Recordings were between 9 and $33 \mathrm{~s}$ long (mean 25.6s) depending on co-operation of the child and the ability of the EHTF to acquire an acceptable recording. The recordings could be started and stopped on the laptop or via a touch button on the videootoscope. The recordings were saved onto a laptop as MOV- or WMV-files (Macbook Pro and PC) and ranged from 0.85 to $7.61 \mathrm{MB}$ size $($ mean $=3.6 \mathrm{MB})$.

\subsection{Remote assessments of video recordings}

After completion of onsite data collection, the video-otoscopy recordings and the short case histories (left/right ear, case number, recorded symptoms) were anonymised and uploaded to a server, using a free web-based hosting service (Dropbox). Recordings were assigned random numbers by an independent investigator at the University of Pretoria, Pretoria, South Africa, before they were downloaded to the University of Umeå, Umeå, Sweden. The first remote evaluation was performed four weeks after the initial onsite data collection, and recordings were again assigned random numbers before the second remote evaluation after eight weeks. The delay in remote assessment was included to counter the possibility of a memory effect for onsite diagnoses and OMGRADE gradings made. The second remote assessment four weeks after the first one also allowed for assessment of intra-rater correspondence.

The otologist who did the original gold standard otomicroscopy and a general practitioner (GP) experienced in otoscopy ( $>15$ years of pratise) completed the remote assessments. Assessments were made separately and were blinded to the randomized numbering of recordings to avoid bias.

The analyses of the video recordings were made using a 24 inch Apple LED Cinema Display connected to a Macbook Pro. The evaluation using the modified OMGRADE-scale steps were individually logged onto separate electronic Excel spreadsheets that were uploaded to the Dropbox server once completed. The independent investigator managed the server.

If any of the examiners considered the video recording to be of insufficient quality for an OMGRADE assessment (obstructing wax, low image quality or poor view of the TM) at any of the remote assessments, the diagnosis was classified as 'not possible to determine' (NPD) and excluded from all further analyses.

\subsection{Data analysis}

Reliability of the OMGRADE scale was determined by investigating the agreement between reviewer gradings at four and eight weeks using Cohen's kappa. Weighted kappa statistic $(\kappa)$ was used to quantify "strength of agreement" (or diagnostic concordance) based upon the range in which kappa statistic matches, for explanation see Tables 3, 4 and 6. The OMGRADE scale can be treated as an ordinal scale and therefore the weighted kappa was more appropriate to compensate for the proximity of gradings.

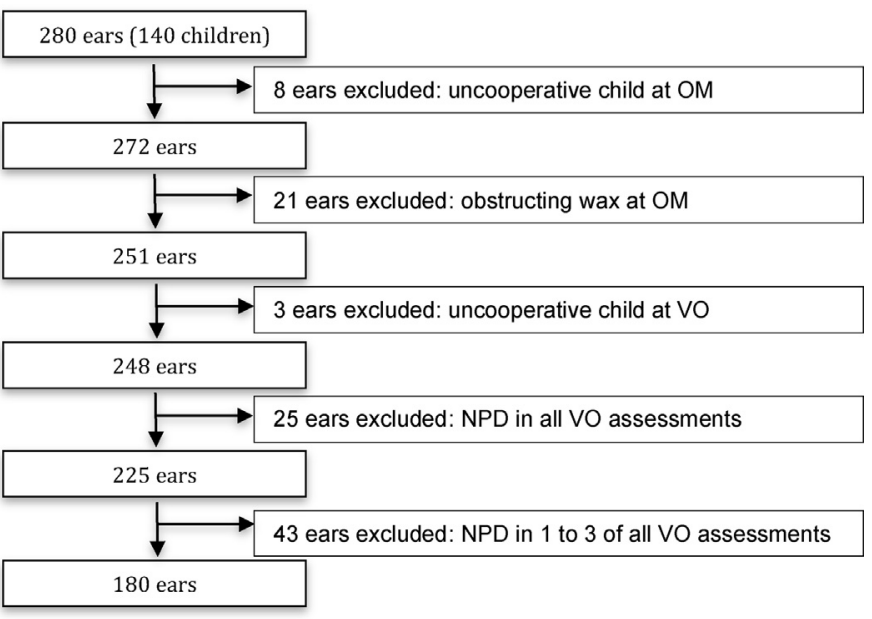

Fig. 2. Excluded ears and reasons for exclusion. 
Table 2

Distribution of OMGRADE gradings at all assessments. Percentages in brackets.

\begin{tabular}{|c|c|c|c|c|c|}
\hline \multirow[t]{3}{*}{ OMGRADE } & \multirow{3}{*}{$\begin{array}{l}\text { OM } \\
\text { Otologist }\end{array}$} & \multicolumn{4}{|c|}{ Video-otoscopy assessments } \\
\hline & & \multicolumn{2}{|l|}{ Otologist } & \multicolumn{2}{|l|}{ GP } \\
\hline & & 4 weeks & 8 weeks & 4 weeks & 8 weeks \\
\hline 0 & $146(81.1 \%)$ & $133(73.9 \%)$ & $144(80 \%)$ & $77(42.8 \%)$ & $90(50 \%)$ \\
\hline $1 \mathrm{R}$ & $8(4.4 \%)$ & $14(7.8 \%)$ & 7 (3.9\%) & $75(41.7 \%)$ & $65(36.1 \%)$ \\
\hline $1 \mathrm{~F}$ & $0(0 \%)$ & $1(0.6 \%)$ & $1(0.6 \%)$ & $1(0.6 \%)$ & $1(0.6 \%)$ \\
\hline $1 \mathrm{RF}$ & $3(1.7 \%)$ & $2(1.1 \%)$ & $1(0.6 \%)$ & $0(0 \%)$ & $1(0.6 \%)$ \\
\hline $2 \mathrm{OF}$ & $1(0.6 \%)$ & $3(1.7 \%)$ & $3(1.7 \%)$ & $5(2.8 \%)$ & $3(1.7 \%)$ \\
\hline 3 & $13(7.2 \%)$ & $13(7.2 \%)$ & $11(6.1 \%)$ & $13(7.2 \%)$ & $10(5.6 \%)$ \\
\hline 4 & $0(0 \%)$ & $0(0 \%)$ & $0(0 \%)$ & $0(0 \%)$ & $0(0 \%)$ \\
\hline $5 B$ & $0(0 \%)$ & $0(0 \%)$ & $0(0 \%)$ & $0(0 \%)$ & $0(0 \%)$ \\
\hline $5 C$ & $0(0 \%)$ & $0(0 \%)$ & $0(0 \%)$ & $0(0 \%)$ & $1(0.6 \%)$ \\
\hline$t-6^{a}$ & $9(5 \%)$ & $14(7.8 \%)$ & $13(7.2 \%)$ & $9(5 \%)$ & $9(5 \%)$ \\
\hline
\end{tabular}

Abbreviations: OM: otomicroscopy; GP: general practitioner.

a Temporary scale step 6 in OMGRADE $=$ CSOM.

Weighted kappa was also used to calculate concordance of OMGRADE otomicroscopy by the otologist, and video-otoscopy assessments by the otologist and GP, together with inter-rater and intra-rater agreement on the video-otoscopy assessments were calculated.

For ears where poor agreement was noted the data was further examined to identify possible causes for the disagreement and to verify if any scale step in the OMGRADE may have caused disagreement between examiners.

The diagnostic validity of the OMGRADE scale was then assessed by calculating sensitivity and specificity from the otologists assessments of diagnoses at otomicroscopy as compared with video-otoscopy gradings. The calculations were done to differentiate abnormal ears from normal ears. All ears graded as t-6 (CSOM) by the otologist on otomicroscopy were excluded from this calculation. The OMGRADE scale steps at video-otoscopy analysis by the otologist were re-grouped as follows: steps 0 and $1 \mathrm{R}$ as "normal" and steps 1F, 1RF, 2OF and 3 as "OME". The otologists assessments of diagnosis at otomicroscopy were defined as the gold standard. Furthermore, a grouping of OMGRADE according to otitis media diagnosis was made. Step 0 and $1 \mathrm{R}$ were grouped as "normal", steps $1 \mathrm{~F}, 1 \mathrm{RF}, 2 \mathrm{OF}$ and 3 as "OME", steps 4, 5B and $5 \mathrm{C}$ as "AOM" and finally the temporary step t-6 as "CSOM". These groupings were then compared to otologist's onsite diagnosis and kappa values for agreement were calculated.

Data analysis of frequencies and cross-tabulations were made using SPSS. An online kappa-calculator was used - http://www. statstodo.com/CohenKappa_Pgm.php - together with Excel and SPSS. An online calculator was also used for calculations of sensitivity and specificity - http://www.medcalc.org/calc/diagnostic_test.php.

Table 3

Agreement between asynchronous grading of video-otoscopic recordings as compared with onsite "gold standard" otomicroscopy grading using OMGRADE.

\begin{tabular}{llll}
\hline & & Weighted kappa $^{\mathrm{a}}$ & 95\% confidence interval \\
\hline \multirow{2}{*}{ Week 4} & Otologist & 0.73 & $0.60-0.85$ \\
& GP & 0.65 & $0.55-0.74$ \\
Week 8 & Otologist & 0.77 & $0.64-0.89$ \\
& GP & 0.71 & $0.63-0.80$ \\
\hline
\end{tabular}

a Kappa statistic. Quantification of "strength of agreement" based upon the range in which kappa statistic matches: "poor agreement" $\kappa<0.00$, "slight agreement" $\kappa=0.01-0.20$, "fair agreement" $\kappa=0.21-0.40$, "moderate agreement" $\kappa=0.41-0.60$, "substantial agreement" $\kappa=0.61-0.80$, "almost perfect agreement" $\kappa=0.81-100$.

\section{Results}

\subsection{Study population}

Of the 140 children enrolled in the study, partial or complete removal of cerumen was required in $36 \%$ of the children $(23.5 \%$ of ears). 17 children reported one or more symptoms during a two-week period before participation. Three of these children reported all symptoms and three reported earache together with discharge. Earache was reported for 10 children whilst previous or on-going ear discharge from one or both ears was reported for seven children. A noticeable hearing loss during the same time period was reported for eight children. Eight of the 17 children who reported symptoms were diagnosed as normal in both ears.

Four children (8 ears) were excluded due to non-compliance at the otomicroscopic examination and a further 21 ears were excluded due to obstructing wax that could not be removed. During video-otoscopy two children were uncooperative and no recordings could be made from three ears. A further 68 ears were excluded from analysis because one or more of the video-otoscopy recordings were regarded as insufficient for assessment of the TM status by any of the examiners (otologist and/or GP). After all exclusions (Fig. 2) 180 ears remained in the study for comparison.

\subsection{Otomicroscopic TM evaluation (gold standard)}

Onsite otomicroscopic diagnosis by the otologist revealed 151 normal ears (84\%), 20 OME ears (11\%) and nine ears with CSOM (5\%). No ears with AOM were found in the ears included for analysis. The otologists otomicroscopic evaluations according to the OMGRADE scale are shown in Table 2.

Table 4

Inter- and intra-rater agreement of asynchronous grading using OMGRADE on video-otoscopic recordings.

\begin{tabular}{llll}
\hline & & Weighted kappa $^{\mathrm{a}}$ & $95 \%$ confidence interval \\
\hline \multirow{2}{*}{ Inter-rater } & 4 weeks & 0.68 & $0.58-0.78$ \\
& 8 weeks & 0.72 & $0.63-0.81$ \\
& & & \\
Intra-rater & Otologist & 0.87 & $0.80-0.95$ \\
& GP & 0.85 & $0.79-0.90$ \\
\hline
\end{tabular}

a Kappa statistic. Quantification of "strength of agreement" based upon the range in which kappa statistic matches: "poor agreement" $\kappa<0.00$, "slight agreement" $\kappa=0.01-0.20$, "fair agreement" $\kappa=0.21-0.40$, "moderate agreement" $\kappa=0.41-0.60$, "substantial agreement" $\kappa=0.61-0.80$, "almost perfect agreement" $\kappa=0.81-100$. 
Table 5

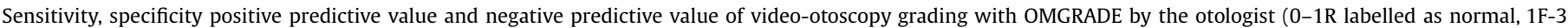
labelled as OME) at week four and eight compared to otologist's otomicroscopic diagnoses normal or OME used as "gold standard".

\begin{tabular}{|c|c|c|c|c|c|c|c|}
\hline & \multicolumn{2}{|c|}{ Otomicroscopic grading } & \multicolumn{2}{|c|}{ Video-otoscopic grading week 4} & \multicolumn{2}{|c|}{ Video-otoscopic grading week 8} & \multirow{2}{*}{$\begin{array}{l}\text { Total } \\
\%\end{array}$} \\
\hline & $\%$ & $95 \% \mathrm{CI}$ & $\%$ & $95 \% \mathrm{CI}$ & $\%$ & $95 \% \mathrm{CI}$ & \\
\hline Sensitivity & 85 & $62-97$ & 70 & $46-88$ & 65 & $45-85$ & 68 \\
\hline Specificity & 100 & $97-100$ & 97 & $92-99$ & 98 & $94-100$ & 97 \\
\hline Positive predictive value & 100 & $80-100$ & 74 & $49-91$ & 81 & $54-96$ & 77 \\
\hline Negative predictive value & 98 & $94-100$ & 96 & $91-98$ & 95 & $91-98$ & 96 \\
\hline
\end{tabular}

Disease prevalence $12 \% . \mathrm{CI}=95 \%$ confidence interval.

Table 6

Agreement between diagnosis at onsite otomicroscopy by otologist and videootoscopic assessments with the grouped OMGRADE scale steps ${ }^{\mathrm{a}}$ at week four and eight.

\begin{tabular}{llll}
\hline & & Weighted kappa $^{\mathrm{b}}$ & $95 \% \mathrm{CI}$ \\
\hline Week 4 & Otologist & 0.75 & $0.60-0.90$ \\
& GP & 0.80 & $0.68-0.92$ \\
Week 8 & Otologist & 0.78 & $0.64-0.93$ \\
& GP & 0.78 & $0.74-0.95$ \\
\hline
\end{tabular}

Abbreviations: $\mathrm{CI}$ : $95 \%$ confidence interval.

a Groupings: 0,1R as normal; $1 \mathrm{~F}, 1 \mathrm{RF}, 2 \mathrm{OF}$ and 3 as OME; 4, 5B and 5C as AOM; t-6 as CSOM.

b Kappa statistic. Quantification of "strength of agreement" based upon the range in which kappa statistic matches: "poor agreement" $\kappa<0.00$, "slight agreement" $\kappa=0.01-0.20$, "fair agreement" $\kappa=0.21-0.40$, "moderate agreement" $\kappa=0.41-0.60$, "substantial agreement" $\kappa=0.61-0.80$, "almost perfect agreement" $\kappa=0.81-100$.

Using the OMGRADE scale the otologist classified 146 TMs as grade 0 , which are normal ears, as compared with 151 normal ears using otologist diagnoses. Eight TMs were graded as $1 \mathrm{R}$, which corresponds with a transparent and only slightly retracted TM. TMs graded as $1 \mathrm{~F}, 1 \mathrm{RF}, 2 \mathrm{OF}$ and 3 were found in 17 ears as compared with 20 ears diagnosed with OME using traditional diagnoses. No TM was graded as OMGRADE 4, 5B or 5C, corresponding to AOM (bulging TM, bullous TM or a wet and perforated ear drum).

Nine of the TMs were graded as grade $6(t-6)$, indicating CSOM. The same number of CSOM ears was found with traditional diagnoses.

\subsection{Remote evaluations of video-otoscopic recordings}

The distribution of OMGRADE gradings made remotely by the otologist and GP after four and eight weeks (Table 2) showed that examiners agreed completely between these sessions when compared to the gold standard of otomicroscopy in 80 of the 180 ears (44.4\%). In 6 ears the examiners also fully agreed at all four sessions (3.9\%) although the otomicroscopy grading was different.

There was substantial agreement between the otologist and GP gradings of video-otoscopy recordings and gold standard otomicroscopy (Table 3). Inter-rater agreement of video-otoscopy evaluations also showed substantial agreement, whilst intra-rater agreement was almost perfect (Table 4).

Low agreement was evident between the examiners for OMGRADE scale step $1 \mathrm{R}$. This scale step was identified by the otologist in eight ears at otomicroscopy and in 14 and 7 ears respectively at the four and eight week otoscopy assessments. The GP identified scale step $1 \mathrm{R}$ in 75 and 65 ears, respectively at the four and eight week's assessments.

At otomicroscopy, the otologist diagnosed 9 ears as t-6 (CSOM), but at the video-otoscopy assessments at four and eight weeks the otologist found 14 and 13 ears, respectively with CSOM. Further analysis showed that the increase in t-6 (CSOM) diagnoses on video-otoscopy (5 and 4 ears at four and eight weeks respectively) were originally classified as normal (grade 0 ) on original onsite otomicropscopy.

\subsection{Diagnostic validity of the omgrade scale}

The calculations of sensitivity and specificity on groupings of video-otoscopy diagnoses by the otologist at four and eight weeks as compared with the otologists diagnoses at otomicroscopy are shown in Table 5.

Calculation on agreement between diagnoses made by otologist onsite compared to the OMGRADE scale steps grouped into diagnoses showed substantial to almost perfect kappa values (Table 6).

\section{Discussion}

This study demonstrates that the OMGRADE scale can be used as a grading tool by an otologist and a GP to assess the TM in remote assessments over the internet using video-otoscopy recordings. Video-otoscopic recordings acquired by an EHTF allowed for remote assessment at a later stage with close concordance to onsite otomicroscopy assessment by an experienced otologist (gold standard). Furthermore, the study demonstrates that the OMGRADE scale can be used in an unselected population of children to detect normal as well as pathological TMs. The OMGRADE scale steps corresponded to onsite diagnosis made by an otologist using otomicroscopy. Despite being developed primarily for AOM the OMGRADE scale provided accurate detection of OME and, with further development, likely also for CSOM.

In the current study population a low number of pathological ears were identified, which is to be expected in an unselected population. This is a limitation of our study that contributed to large $95 \%$ confidence intervals. This may also, to some extent, be due to the fact that the study did not include children below the age of two years in which otitis media and AOM, in particular, is most common.

In diagnosing AOM and OME an evaluation of TM mobility is important to assess middle ear fluid. However, no evaluation of TM mobility was made due to lack of equipment (tympanometer and pneumatic otomicroscopy) and may impair the diagnostic certainty for OME. It is known that otomicroscopy alone has a high sensitivity (87-91\%) and specificity (89-93\%) in detecting middle ear effusion [20,21]. Documentation of case history symptoms in the current study was minimal and may not have corresponded to current criteria for diagnosing AOM [22]. Due to the absence of ears diagnosed with AOM in this study, the reliability of AOM grading and diagnosis using the OMGRADE scale could not be assessed. Despite this, the OMGRADE's reliability could be evaluated in respect of grading and diagnosis of paediatric ears without pathology and with other middle ear pathology

Clinical evaluations that are based on visual findings are difficult to reach consensus about and may result in some dispute 
in interpretation of findings. Kappa values found in our study were, however promising in comparison to other studies. Shaik et al. [12] showed low agreement on assessing colour of TM in still images with mean kappa values of 0.32 and 0.33 . The agreement on translucency and position of TM was higher (mean kappa values 0.55 and 0.56 , respectively). Patricoski et al. [23] evaluated the TM for retraction, middle ear fluid and ventilation tube patency. They demonstrated kappa values for onsite examination on "tube in" of 0.93 , middle ear fluid of 0.14 and retraction of TM 0.49 . An image based grading scale gives visual references for the reviewers to adhere to, which may increase the concordance.

The main reason for disagreement on TM assessments in the current study was related to the OMGRADE scale step $1 \mathrm{R}$, namely the slightly, and most likely, transiently retracted but transparent TM, which would be regarded as a clinically normal TM. The GP rated TM's as $1 \mathrm{R}$ five to eleven times more often than the otologist did and this may be due to two reasons. Firstly, the discrepancy in $1 \mathrm{R}$ may be caused by insufficient calibration of the otologist and GP before the study. Secondly, scale step $1 \mathrm{R}$ appears to be difficult to assess leading to discrepancies in the assessments. Grade $1 \mathrm{R}$ is not regarded as clinically relevant to identify in otitis media and, therefore, a revision of the OMGRADE will be proposed. In the revised OMGRADE-scale the scale step $1 \mathrm{R}$ would probably be transferred to grade 0 (normal TM with or without slight retraction of no clinical pathological significance). We have performed a recalculation of our findings transferring all grade 1 R's to grade 0 , resulting in increased kappa values. Weighted kappa values increased for the GP with respect to concordance between otomicroscopy versus video-otoscopy assessments, ranging from 0.80 to 0.84 , and in inter-rater agreement from 0.79 to 0.83 . Even the kappa values for intra-rater agreement increased to 0.90 for both otologist and GP. This leads us to conclude that a revision of the OMGRADE scale would improve the reliability of the scale. The addition of a scale step to indicate CSOM would also increase the usability of the scale for all forms of otitis media. The supplementary scale step named t-6 was used in our study to identify ears with CSOM. We found concordance between CSOM diagnosis and the grading of $\mathrm{t}-6$ to be adequate.

Validated tools to grade and diagnose otitis media and AOM in particular are sparse. McCormick et al. [9] have created the OS-8 that focuses more on TM redness compared to the OMGRADE scale. The OS-8 also lacks the classification of a wet and contourless/ perforated TM, described as "chagrinated" in the OMGRADE scale. Different types of scoring systems, as opposed to image based grading scales, have been widely used in previous research. These non-validated scoring systems give the reddened TM the same weight of importance as the bulging of TM. Casey et al. [7] presented a 10-point scoring system that included symptom scores as well as otoscopic sign scores. They found sensitivity and specificity to be $87 \%$ and $97 \%$, respectively. However, that study was performed in a selected population of children diagnosed with AOM compared to the unselected population with low disease prevalence in our study.

A reliable standardized grading scale and a diagnostic guide such as the OMGRADE scale could be of significance in clinical practice as well as in otitis media research to ensure more uniform, standardized assessments. Future research should focus on the clinical value of a revised OMGRADE scale in combination with assessment of middle ear effusion as a diagnostic guide for GP's and registrars in general practice, and for ear-, nose- and throat specialists and pediatricians engaged in otology. It could also serve as an aid in teaching of medical students. The present study demonstrates that it could be useful and reliable to assess TMs in telemedicine facilities with remote assessments of videootoscopic recordings. Furthermore, OMGRADE could be used in research for grading the severity of AOM or to evaluate the effect of treatment in intervention studies. Unexpectedly, this study also indicated that video-otoscopic recordings might, at times, provide a more reliable diagnosis as compared to onsite examinations using otomicroscopy. The relatively high number of video-otoscopic recordings that were not possible to assess is, however, something that needs to be addressed in future studies. A more thorough training period of the video-otoscopist is to be recommended.

In conclusion, we found that the OMGRADE scale, initially designed for AOM, can be used to distinguish the normal TM from pathological TMs in OME in an unselected population of children. The OMGRADE scale, in its present form, can be used as a tool to assess the TM in remote assessments over the internet using video-otoscopy recordings performed by a clinic facilitator without prior health care training. However, a revision of the OMGRADE scale is proposed to further improve its usability and reliability, and also to include CSOM.

\section{References}

[1] L. Monasta, L. Ronfani, F. Marchetti, M. Montico, L. Vecchi Brumatti, A. Bavcar, et al., Burden of disease caused by otitis media: systematic review and global estimates, PLoS One 7 (2012) e36226.

[2] J.J. Fagan, M. Jacobs, Survey of ENT services in Africa: need for a comprehensive intervention, Global Health Action 2 (2009).

[3] A.C. Smith, N.R. Armfield, W.I. Wu, C.A. Brown, C. Perry, A mobile telemedicine-enabled ear screening service for Indigenous children in Queensland: activity and outcomes in the first three years, J. Telemed. Telecare 18 (2012) 485-489.

[4] W. Swanepoel de, D. Koekemoer, J. Clark, Intercontinental hearing assessment a study in tele-audiology, J. Telemed. Telecare 16 (2010) 248-252.

[5] S.M. Chandler, S.M. Garcia, D.P. McCormick, Consistency of diagnostic criteria for acute otitis media: a review of the recent literature, Clin. Pediatr. 46 (2007) 99-108.

[6] J.S. Gravel, P. Karma, M.L. Casselbrant, P. Marchisio, A. Andalibi, D. Passali, et al., Recent advances in otitis media. 7. Diagnosis and screening, Ann. Otol. Rhinol. Laryngol 194 (suppl) (2005) 104-113.

[7] J.R. Casey, S. Block, P. Puthoor, J. Hedrick, A. Almudevar, M.E. Pichichero, et al., A simple scoring system to improve clinical assessment of acute otitis media, Clin. Pediatr. 50 (2011) 623-629.

[8] M. Hotomi, N. Yamanaka, T. Samukawa, M. Suzumot, A. Sakai, J. Shimada, et al. Treatment and outcome of severe and non-severe acute otitis media, Eur. J. Pediatr. 164 (2005) 3-8.

[9] D.P. McCormick, K.A. Saeed, C. Pittman, C.D. Baldwin, N. Friedman, D.C Teichgraeber, et al., Bullous myringitis: a case-control study, Pediatrics 112 (2003) 982-986.

[10] R. Satran, E. Leibovitz, S. Raiz, L. Piglansky, J. Press, A. Leiberman, et al., Clinical/ otologic score before and during treatment of acute otitis media, Acta Paediatr. 96 (2007) 1814-1818.

[11] R.H. Schwartz, S.E. Stool, W.J. Rodriguez, K.M. Grundfast, Acute otitis media: toward a more precise definition, Clin. Pediatr. 20 (1981) 549-554.

[12] N. Shaikh, A. Hoberman, P.H. Kaleida, H.E. Rockette, M. Kurs-Lasky, H. Hoover, et al., Otoscopic signs of otitis media, Pediatr. Infect. Dis. J. 30 (2011) 822-826, doi:http://dx.doi.org/10.1097/INF.1090b1013e31822e36637.

[13] T. Lundberg, S. Hellstrom, H. Sandstrom, Development and validation of a new grading scale for otitis media, Pediatr. Infect. Dis. J. 32 (2013) 341-345.

[14] R.H. Eikelboom, M.N. Mbao, H.L. Coates, M.D. Atlas, M.A. Gallop, Validation of tele-otology to diagnose ear disease in children, Int. J. Pediatr. Otorhinolaryngol. 69 (2005) 739-744.

[15] A. Aronzon, A.T. Ross, K. Kazahaya, M. Ishii, Diagnosis of middle ear disease using tympanograms and digital imaging, Otolaryngol. Head Neck Surg. 131 (2004) 917-920.

[16] R.G. Jensen, A. Koch, P. Homoe, Long-term tympanic membrane pathology dynamics and spontaneous healing in chronic suppurative otitis media Pediatr. Infect. Dis. J. 31 (2012) 139-144.

[17] L. Biagio, W. Swanepoel de, A. Adeyemo, J.W. Hall 3rd, B. Vinck, Asynchronous video-otoscopy with a telehealth facilitator, Telemed. J. E Health 19 (2013) $252-258$.

[18] J. Carruthers, Dainfern and diepsloot: environmental justice and environmental history in Johannesburg, South Africa, Environ. Justice 1 (2008) 6.

[19] G.A. Gates, J.O. Klein, D.J. Lim, G. Mogi, P.L. Ogra, M.M. Pararella, et al., Recent advances in otitis media. 1. Definitions, terminology, and classification of otitis media, Ann. Otol. Rhinol. Laryngol. 188 (suppl) (2002) 8-18. 
[20] M.J. Fields, R.S. Allison, P. Corwin, P.S. White, J. Doherty, Microtympanometry, microscopy and tympanometry in evaluating middle ear effusion prior to myringotomy, N. Z. Med. J. 106 (1993) 386-387.

[21] T. Ovesen, P.B. Paaske, O. Elbrond, Accuracy of an automatic impedance apparatus in a population with secretory otitis media: principles in the evaluation of tympanometrical findings, Am. J. Otolaryngol. 14 (1993) 100-104.
[22] A.S. Lieberthal, A.E. Carroll, T. Chonmaitree, T.G. Ganiats, A. Hoberman, M.A. Jackson, et al., The diagnosis and management of acute otitis media, Pediatrics 131 (2013) e964-e999.

[23] C. Patricoski, J. Kokesh, A.S. Ferguson, K. Koller, G. Zwack, E. Provost, et al, A comparison of in-person examination and video otoscope imaging for tympanostomy tube follow-up, Telemed. J. E Health 9 (2003) 331-344. 
Appendix A. Supplementary data

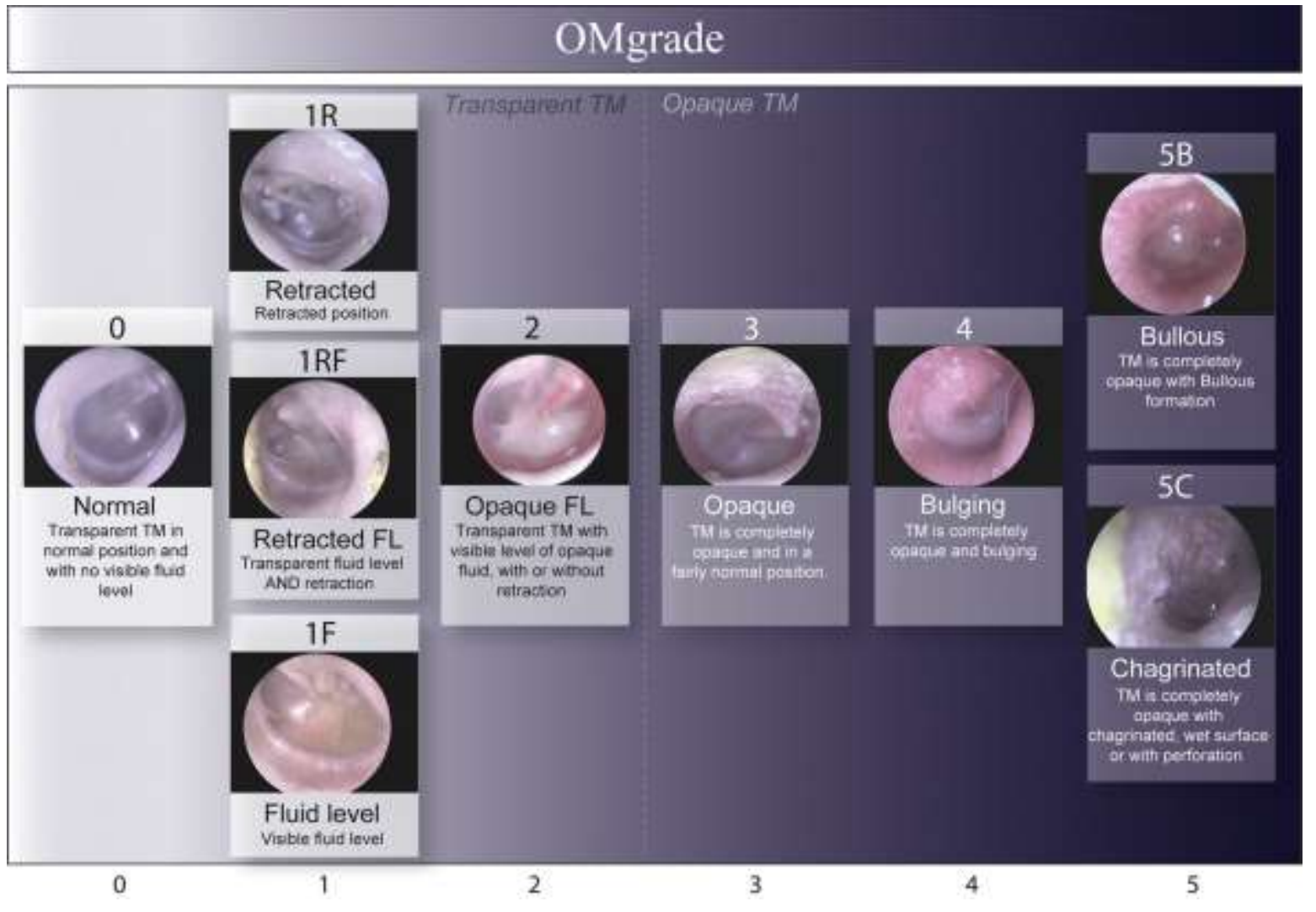

